\title{
Estresse e resiliência em discentes de enfermagem de duas universidades públicas paulistas
}

Stress and resilience in nursing students from two public universities in sao paulo

Estrés y resiliencia en estudiantes de enfermería de dos universidades publicas paulistas

\author{
Fernando Oliveira de Souza', Rodrigo Marques da Silva ${ }^{\text {II }}$, Ana Lúcia Siqueira Costa ${ }^{\mathrm{III}}$ \\ Fernanda Carneiro Mussi' ${ }^{\mathrm{V}}$, Carla Chiste Tomazoli Santos ${ }^{\mathrm{v}}$, Osmar Pereira dos Santos ${ }^{\mathrm{VI}}$
}

\begin{abstract}
Resumo: Objetivo: verificar a relação entre estresse e resiliência em discentes de enfermagem de duas universidades públicas do Estado de São Paulo. Método: trata-se de um estudo transversal realizado em março de 2016 junto a 117 discentes de enfermagem. Aplicaram-se um Formulário para caracterização acadêmica e demográfica, o Instrumento para Avaliação do Estresse em Discentes de Enfermagem e a Escala de Resiliência de Wagnilde Young. Analisaram-se os dados no Statistical Package for Social Sciences, versão 10.0. Resultados: observou-se predomínio de discentes com médio nível de estresse. O gerenciamento do tempo e as atividades teóricas representaram alto nível de estresse para $23,9 \%$ e $20,5 \%$ da população. $11,1 \%$ apresentaram muito alto estresse relacionado ao Ambiente. Os níveis de resiliência foram reduzidos (51\%). Não houve correlação significativa entre estresse e resiliência. Conclusão: o ambiente de formação em enfermagem apresenta potencial para o adoecimento dos discentes, embora parte deles já apresente resiliência moderada.
\end{abstract}

Descritores: Enfermagem; Estudantes de enfermagem; Resiliência psicológica

Abstract: Aim: to verify the relation between stress and resilience in nursing students from two public universities in the State of São Paulo. Method: this is a cross-sectional study conducted in March 2016 with 117 nursing students. We applied academic and sociodemographic forms, the instrument for nursing students stress

\footnotetext{
${ }^{\text {I }}$ Enfermeiro. Faculdade de Ciências e Educação Sena Aires. Valparaíso de Goiás, GO, Brasil. E-mail: fernandobration2015@gmail.com ORCID: https://orcid.org/0000-0003-1178-5387

II Enfermeiro. Doutor em Enfermagem. Faculdade de Ciências e Educação Sena Aires. Valparaíso de Goiás, GO, Brasil. E-mail: marquessm@hotmail.com ORCID: https://orcid.org/0000-0003-2881-9045

${ }^{\text {III }}$ Enfermeira. Doutora em Enfermagem. Professora livre-docente do Departamento de Enfermagem Médico-cirúrgica da Escola de Enfermagem da Universidade de São Paulo. São Paulo, SP, Brasil. E-mail: anascosta@usp.br ORCID: https://orcid.org/0000-0001-7830-9751

IV Enfermeira. Doutora em Enfermagem. Universidade Federal da Bahia (UFBA). Salvador, BA, Brasil. E-mail: femussi@uol.com.br ORCID: https://orcid.org/0000-0003-0692-5912

${ }^{v}$ Fisioterapeuta. Mestre em Fisioterapia. Docente da Faculdade de Ciências e Educação Sena Aires. Valparaíso de Goiás, GO, Brasil. E-mail: carlatomazolisantos@hotmail.com ORCID: https://orcid.org/0000-0002-5729-7904

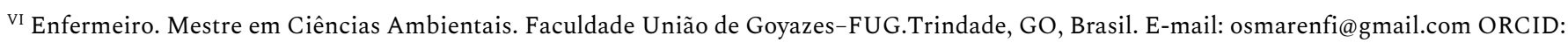
https://orcid.org/0000-0002-7962-622X
} 
Estresse e resiliência em discentes de enfermagem de duas universidades públicas paulistas |2

assessment, and the Wagnild and Young's resilience scale. Data were analyzed in Statistical Package for Social Sciences, version 10.0. Results: we found a predominance of moderately stressed students. Time management and theoretical activities meant high stress level for $23,9 \%$ and $20,5 \%$ of the sample. Also, $11,1 \%$ showed a very high stress level related to the work environment. Levels of resilience were low (51\%). There was not a significant correlation between stress and resilience. Conclusion: nursing education setting shows potential for students' illness, although part of them already show moderate resilience.

Descriptors: Nursing; Students, Nursing; Resilience, Psychological

Resumen: Objetivo: verificar la relación entre estrés y resiliencia en discentes de enfermería de dos universidades públicas del Estado de São Paulo. Método: se trata de un estudio transversal realizado en marzo de 2016 con 117 discentes de enfermería. Se aplicó un formulario para caracterización académica y demográfica, el Instrumento para Evaluación de Estrés en discentes de enfermería y la escala de resiliencia de Wagnild y Young. Los datos fueron evaluados en el Statistical Package for Social Sciences, versión 10.0. Resultados: se observó el predominio de discentes con nivel medio de estrés. El gerenciamiento del tiempo y las actividades teóricas representaron alto nivel de estrés para $23,9 \%$ y $20,5 \%$ de la población. $11,1 \%$ presentaron muy alto el nivel de estrés relacionado al Ambiente. Los niveles de resiliencia fueron bajos (51\%). No hubo correlación significativa entre estrés y resiliencia. Conclusión: el ambiente de formación en enfermería presenta potencial para el padecimiento de los discentes, aunque parte de ellos ya presente moderada resiliencia.

Descriptores: Enfermería; Estudiantes de enfermería; Resiliencia psicológica

\section{Introdução}

No panorama atual, observa-se uma pressão social cada vez maior para que os seres humanos estejam sempre se qualificando e, no ambiente de ensino superior, há uma crescente exigência porque agrega o amadurecimento do pensamento, a aquisição do conhecimento e a busca por um trabalho na área de graduação. Os fatores externos à educação acadêmica, como a experiência de frustação e amadurecimento, as dificuldades financeiras, a distância dos familiares quando o discente mora na cidade apenas para estudo e a necessidade de utilizar um ou mais meios de transporte públicos, são constantemente vividos pelos universitários. ${ }^{1-2}$ Além disso, destacam-se questões como a entrada na vida adulta, os relacionamentos profissionais e pessoais, as pressões socioeconômicas e o mercado de trabalho. ${ }^{1-2}$ A associação dos componentes externos com aqueles próprios da vida acadêmica, como o maior grau de exigência curricular, as atividades extracurriculares e a necessidade de lidar com o sofrimento do outro e 
com a responsabilidade para com a vida humana, pode sobrecarregar os recursos de adaptação dos discentes, levando-os ao estresse. ${ }^{2}$

Oestresse despertou o interesse médico primeiro em Hipócrates (470-377 a.C.) e, posteriormente em Cannon. ${ }^{3}$ Ele foi o responsável por desenvolver o conceito de homeostase capacidade de auto regulação dos sistemas presentes no corpo humano - e por relacionar o estresse com uma interação corpo e mente. No campo das ciências biológicas, no século XX, a conceituação de estresse foi desenvolvida por Hans Selye e a ênfase se dá nas manifestações neuroendócrinas que ocorrem no indivíduo mediante estímulos internos ou externos. ${ }^{3}$ No modelo interacionista, estresse é conceituado como qualquer estímulo que demande do ambiente externo ou interno e que taxe ou exceda as fontes de adaptação de um indivíduo ou sistema social. ${ }^{4}$ No contexto acadêmico, o estresse ocorre quando o estudante percebe as demandas como excessivas aos recursos adaptativos que possui. ${ }^{2}$

Por consequência desse fenômeno, é possível que manifestações físicas e psicológicas estejam presentes, dentre as quais, a cefaleia, a depressão e a ansiedade que podem comprometer o desempenho acadêmico e o relacionamento entre amigos e familiares. ${ }^{5}$ Além disso, é possível que o estresse leve à desfechos negativos como os sintomas depressivos; as alterações na qualidade do sono e qualidade de vida, conforme verificado em estudos anteriores..$^{5-6}$ A exemplo disso, destaca-se pesquisa realizada com 88 discentes de enfermagem de uma universidade pública de São Paulo, no qual se verificou correlação estatisticamente significativa entre estresse e sinais indicativos de depressão, especialmente de depressão grave. ${ }^{5}$

Contudo, mesmo expostos ao estresse no ambiente acadêmico universitário,alguns discentes apresentam menores níveis de estresse na experiência comas adversidades. A resiliência consiste na habilidade de se recuperar das adversidades e se adaptar positivamente a situações da vida. ${ }^{7-8}$ Ela advém de uma somatória de fatores externos e internos, incluindo aspectos emocionais, socioculturais, ambientais e cognitivos que interatuam e permitem que o 
Estresse e resiliência em discentes de enfermagem de duas universidades públicas paulistas $\mid 4$

indivíduo enfrente, vença, fortaleça e se transforme das adversidades e estresse. ${ }^{7}$ Assim, por ser resiliente, espera-se que o indivíduo apresente menores níveis de estresse e, por consequência, menor chance de desfechos como Síndrome de Burnout, sintomas depressivos, estresse e ansiedade..$^{7-8}$

No entanto, apesar de existirem estudos sobre o estresse dos discentes, poucos abordam esse fenômeno no ensino superior brasileiro, especialmente em relação à presença de resiliência aplicada a estes. ${ }^{1-2 ;-8}$ Nesse sentido, a partir da avaliação da personalidade resiliente e de sua relação com estresse acadêmico, será possível desenvolver e adotar ações para promovê-la e, então, minimizar os riscos à saúde do discente..$^{7-8}$

Como hipótese, defende-se que haja correlação significativa entre estresse e resiliência, de forma o aumento dos níveis de resiliência implique menor nível de estresse acadêmico. Como questão de pesquisa, define-se: Há associação significativa entre estresse e resiliência nos discentes universitários de enfermagem?

Nesse sentido, esse estudo teve como objetivo verificar a relação entre estresse e resiliência em discentes de enfermagem de duas universidades públicas do Estado de São Paulo.

\section{Método}

Trata-se de um estudo transversal e com abordagem quantitativa, realizado com todos os discentes de enfermagem do primeiro ano de uma universidade Estadual e outra Federal, ambas localizadas no Estado de São Paulo, que aceitaram voluntariamente participar da pesquisa mediante a assinatura do Termo de Consentimento Livre e Esclarecido (TCLE). Foram incluídos discentes regulamente matriculados no primeiro ano de cada instituição e maiores de 18 anos. Foram excluídos aqueles que, no período de coleta, estavam impedidos de realizar todas as disciplinas do semestre seguinte devido a reprovações no semestre anterior.

A coleta dos dados ocorreu por meio da utilização de um protocolo de pesquisa aplicado em março de 2016. Esse protocolo foi composto pelos seguintes instrumentos: Formulário para 
5 | Souza fO, Silva RM, Costa ALS, Mussi FC, Santos CCT, Santos OP

caracterização acadêmica; Instrumento para Avaliação do Estresse em Discentes de Enfermagem (AEEE) $)^{3}$ e Escala de Resiliência de WagnildeYoung. ${ }^{9}$

O Formulário para caracterização da população abordou as seguintes variáveis sociodemográficas: data de nascimento, sexo, se possui filhos, situação conjugal, com quem reside, realização de atividades de lazer, prática de esportes; e acadêmicas: meio de transporte, número de disciplinas cursadas no semestre atual, carga horária no semestre atual, número diário de horas de estudo, realização e tipo de atividades extracurriculares exercidas e atividade de trabalho.

O Instrumento para Avaliação de Estresse em Discentes de Enfermagem (AEEE) foi proposto por Costa e Polak ${ }^{3}$ e é composto por 30 itens agrupados em seis domínios: Realização das atividades práticas (Itens 4,5,7,9,12 e 21); Comunicação profissional (Itens 6,8,16 e 20); Gerenciamento do tempo (Itens 3,18,23, 26 e 30); Ambiente (Itens 11,22,24 e 29); Formação profissional (Itens 1,15,17,19,25 e 27); Atividade teórica (Itens 2,10,13,14 e 28). Os itens apresentam-se em escala tipo Likert de quatro pontos em que: zero - "não vivencio a situação"; um- "não me sinto estressado com a situação"; dois - "me sinto pouco estressado com a situação"; e três- “me sinto muito estressado com a situação”. ${ }^{3}$ Para identificação da intensidade de estresse por fator do AEEE, foram utilizados quartis de risco, conforme definido pelo autor do instrumento. ${ }^{3}$ Para verificar o nível de estresse geral por discente, foi realizada a soma dos escores atribuídos aos 30 itens do instrumento, sendo comparados os escores de estresse obtidos no início e no final do primeiro ano letivo de curso. Os valores de Alfa obtidos para os domínios do AEEE no processo de validação foram respectivamente: 0,806 (Realização de Atividades Práticas), 0,768 (Comunicação Profissional), 0,717 (Gerenciamento do Tempo), 0,866 (Ambiente), 0,772 (Formação Profissional), 0,720 (Atividade Teórica). ${ }^{3}$

A escala de resiliência, desenvolvida por Wagnild \& Young junto a mulheres adultas, foi adaptada e traduzida para a realidade brasileira junto a discentes (de ambos os sexos) de escolas 
Estresse e resiliência em discentes de enfermagem de duas universidades públicas paulistas |6

públicas em 2005.9-10 Esse instrumento mede os níveis de adaptação psicossocial positiva em face de eventos de vida importantes. Ele possui 25 itens em escala tipo likert, variando de 1 (discordo totalmente) a 7 (concordo totalmente), distribuídos em 3 fatores, a ser: Resoluções de Ações e Valores (Itens 1,2,6,8,10,12,14,16,18,19,21,23,24 e 25), Independência e determinação (Itens 5,7,9,11,13 e 22) e Autoconfiança e capacidade de adaptação a situações (Itens 3,4,15,17 e 20). ${ }^{9}$ Esses fatores correspondem aos atributos que dão suporte ao enfrentamento dos problemas da vida, incluindo competência nas relações sociais, a capacidade de resolução de problemas, a conquista de autonomia e o sentido ou propósito para a vida e o futuro. ${ }^{9-10}$ Os escores da escala oscilam de 25 a 175 pontos, sendo que quanto maior o escore, mais elevada é a resiliência do sujeito. Um resultado abaixo dos 121 é considerado pelos autores do instrumento como “reduzida resiliência"; entre 121 e 145, como "resiliência moderada”; e acima dos 145, "resiliência elevada". ${ }^{10}$ No processo de validação, o instrumento apresentou boa propriedades psicométricas, com Alfa de Cronbach de 0,80 para a amostra total. ${ }^{9}$

No período de coleta, esses instrumentos foram entregues presencialmente em sala de aula, após autorização e agendamento direto com os docentes das disciplinas a serem ministradas nos cursos de enfermagem, sendo agendada uma data para recolhimento dos mesmos. O recolhimento em sala de aula contou com o auxílio dos representantes de turma e de discentes que se propuseram a auxiliar nesse processo. Além disso, para aumento da adesão à pesquisa, foram enviados individualmente, via e-mail, os resultados individuais aos discentes que participaram da pesquisa.

Para organização e análise dos dados, foi criado um banco de dados no programa Excel (Office 2010) e utilizado o Pacote estatístico Statistical Package for Social Sciences (SPSS) versão 10.0. Os instrumentos foram analisados conforme descrito anteriormente, preconizandose a análise descrita pelos autores dos instrumentos. Para avaliar relações entre os escores de estresse e resiliência, foi utilizado o teste de correlação de Pearson. Os resultados foram 
considerados estatisticamente significantes se $\mathrm{p}<0,05$.O Alfa de Cronbach foi aplicado para análise da confiabilidade dos instrumentos aplicados.

O projeto de pesquisa foi apreciado e aprovado em $1^{\circ}$ de fevereiro de 2016 no Comitê de Ética em Pesquisa (CEP) da instituição pública estadual, sob parecer n¹.363.890. Posteriormente, foi submetida emenda ao CEP para inclusão da instituição publica federal como local de coleta de dados, com aprovação sob parecer $\mathrm{n}^{\circ} 1.400 .103$. Ademais, esse projeto foi submetido ao Serviço de Pesquisa da instituição estadual para que fosse apreciado quanto à viabilidade e mérito, sendo também aprovado pela comissão responsável. Atendendo às Diretrizes e Normas Regulamentadoras de Pesquisas Envolvendo Seres Humanos (Resolução CNS 466/12), foi entregue aos participantes de pesquisa um Termo de Consentimento Livre e Esclarecido junto aos instrumentos, o qual foi assinado em duas vias (uma para o participante e outra para o pesquisador), autorizando a participação voluntária na pesquisa.

\section{Resultados}

Em março, dos 183 alunos matriculados nas duas instituições pesquisadas, 143 aceitaram participar da pesquisa, porém 117 devolveram os instrumentos preenchidos.

$\mathrm{Na}$ análise da confiabilidade dos instrumentos, observa-se Alfa de Cronbach de 0,86 para os 30 itens do AEEE e de 0,85 para os 25 itens da Escala de Resiliência, valores que atestam confiabilidade satisfatória aos instrumentos utilizados. ${ }^{11} \mathrm{Na}$ Tabela 1 , apresenta-se a caracterização sociodemográfica e acadêmica dos discentes de enfermagem nos dois períodos analisados.

Tabela 1- Caracterização sociodemográfica e acadêmica dos discentes de enfermagem. São Paulo, 2016.

\begin{tabular}{lc}
\hline \multicolumn{1}{c}{ Variáveis sociodemográficas* } & n(\%) ou Média $(\mathbf{D P})$ \\
\hline Sexo(Feminino) & $99(84,6 \%)$ \\
Idade & $20,73(4,4)$ \\
Situação Conjugal (Solteiro/Sem companheiro) & $98(83,8 \%)$ \\
Filhos (Não) & $112(95,7 \%)$ \\
Esporte (Não) & $77(65,8 \%)$ \\
\hline
\end{tabular}


Estresse e resiliência em discentes de enfermagem de duas universidades públicas paulistas $\mid 8$

\begin{tabular}{|c|c|c|}
\hline \multicolumn{2}{|l|}{ Lazer (Sim) } & $71(60,7 \%)$ \\
\hline \multicolumn{2}{|c|}{ Variáveis acadêmicas* } & n $(\%)$ ou Média (DP) \\
\hline \multirow{2}{*}{$\begin{array}{l}\text { Tempo gasto até à instituição } \\
\text { (Minutos) }\end{array}$} & Instituição A & $68,3(26,1)$ \\
\hline & Instituição B & $71,7(41,1)$ \\
\hline \multicolumn{2}{|l|}{ Horas de Estudo por dia } & $2,7(1,3)$ \\
\hline \multicolumn{2}{|l|}{ Número de disciplinas cursadas } & $11(2,9)$ \\
\hline \multicolumn{2}{|c|}{ Atividades Extracurriculares (Sim) } & $66(56,4 \%)$ \\
\hline \multicolumn{2}{|c|}{ Possui atividade de Trabalho (Não) } & $109(93,2 \%)$ \\
\hline \multirow[t]{2}{*}{ Carga Horária semestral ${ }^{\star \star}$} & Instituição A & $442,9(160,1)$ \\
\hline & Instituição B & $848,6(253,9)$ \\
\hline
\end{tabular}

${ }^{*}$ As categorias predominantes para cada variável são apresentadas.

${ }^{* *}$ Média obtida a partir dos valores informados pelos alunos.

$\mathrm{Na}$ Tabela 1, verifica-se predomínio de discentes do sexo feminino, jovens (com aproximadamente 20 anos de idade), solteiros, sem filhos, que praticam atividades de lazer, porém não praticam atividadesesportivas. Quanto às variáveis acadêmicas, observa-se que os discentes dispensam aproximadamente 1 hora para chegarem até as instituições de ensino; que não exercem atividades de trabalho, estão matriculados em 11 disciplinas, em média, ao longodo ano letivo, sob uma carga horária semestral média que varia de 442,9 (Instituição A) a 848,6 (Instituição B) e que realizam atividades extracurriculares.

Observa-se predomínio de discentes com médio nível de estresse (72,60\%), seguido por aqueles que relataram alto estresse (17,9\%). Nenhum aluno apresentou muito alto estresse. $\mathrm{Na}$ Figura 1, apresenta-se a porcentagem de níveis de estresse por fator do instrumento de Avaliação de Estresse em Estudantes de Enfermagem-AEEE nos discentes de enfermagem.

Figura 1- Porcentagem dos níveis de estresse por fator do AEEE nos discentes de enfermagem. São Paulo, 2016. 


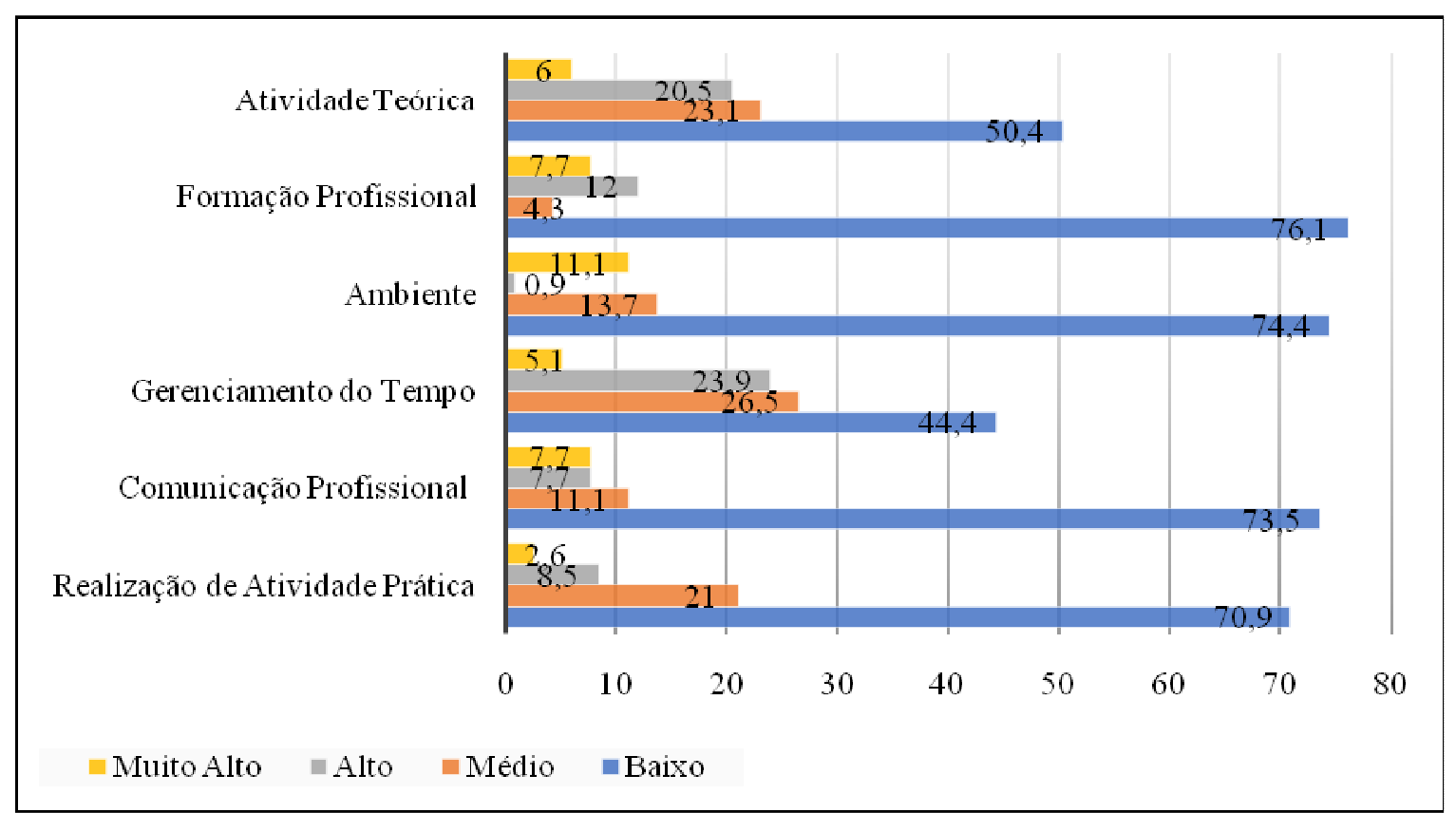

De modo geral, houve predomínio de baixo nível de estresse em todos os domínios, seguido por moderado estresse. No entanto, observa-se que o gerenciamento do tempo e as atividades teóricas representam alto nível de estresse para 23,9\% e 20,5\% dos discentes de enfermagem. Além disso, $11,1 \%$ dos discentes apresentaram muito alto estresse relacionado ao Ambiente.

Verifica-se predomínio de reduzidos níveis de resiliência (51\%) entre os discentes, seguido por níveis moderados (45\%) de resiliência. Além disso, ao analisar asmédias por domínio do instrumento, observa-se que o domínio Ações e Valores (Média= 5,06; Desvio-padrão= 0,84) é o que mais contribui para a referida personalidade entre os discentes. Na Tabela 2, apresenta-se a análise de associação entre o estresse e a personalidade resiliente.

Tabela 2- Análise de associação entre o estresse e a personalidade Resiliente em discentes de enfermagem. São Paulo, 2016.

\begin{tabular}{cccc}
\hline $\mathbf{X}$ & $\mathbf{Y}$ & $\mathbf{r}$ & Valor de $\mathbf{p}$ \\
Resiliência & Estresse & -0.061 & 0.510 \\
Resiliência & RAP & -0.068 & 0.468 \\
Resiliência & CP & -0.149 & 0.108 \\
Resiliência & GT & -0.025 & 0.790 \\
Resiliência & AMB & 0.074 & 0.431 \\
Resiliência & FP & -0.121 & 0.193 \\
Resiliência & AT & 0.058 & 0.535 \\
\hline
\end{tabular}


Estresse e resiliência em discentes de enfermagem de duas universidades públicas paulistas | 10

Nota: RAP-Realização de Atividades Práticas; CP- Comunicação Profissional; GT- Gerenciamento do Tempo; AMB- Ambiente; FP- Formação Profissional; AT- Atividades Teóricas

Conforme tabela acima, observa-se que não houve correlação significativa entre os escores de estresse (geral e por domínio) e os níveis de resiliência entre os discentes de enfermagem avaliados.

\section{Discussão}

Em relação ao perfil dos entrevistados, observou-sepredomínio de mulheres, solteiras, sem filhos e que praticam atividades de lazer. Os discentes geralmente levam 1 hora para chegar à faculdade, não possuem vínculo de trabalho, cursam 11 matérias durante o ano (em média) e participam de atividades extracurriculares. Em pesquisa realizada com 214 estudantes de enfermagem de uma universidade públicado Rio Grande do Sul, verificou-se predomínio de discentes do sexo feminino (89\%), solteiros (97\%) e sem filhos (99\%). A preponderância de mulheres no campo da enfermagem tem caráter histórico dado que a enfermagem, no seu surgimento, era uma profissão exclusivamente feminina. ${ }^{12}$ Hoje, apesar o incremento do número de homens que compõem as turmas em formação nos cursos de enfermagem e nas equipes de enfermagem dos serviços de saúde, ainda há predominância feminina.

Além do trabalho, as discentes mulheres acumulam o trabalho da casa e o cuidado aos filhos, o que pode levar a sobrecarga e ao estresse acadêmico. Nessa perspectiva, é compressível que, na busca por uma maior dedicação a carreira profissional e estabilidade profissional e financeira, muitas mulheres optam por não ter filhos. No caso de estudantes da área de saúde, a maior proximidade com o conhecimento relacionado aos métodos contraceptivos pode contribuir para o sucesso na busca de não ter filhos. ${ }^{12-13}$

Quanto ao estresse por domínio do AEEE, embora com predominância de baixo estresse nos fatores do instrumento, observou-se que o gerenciamento do tempo e as atividades teóricas representam alto nível de estresse para 23,9\% e 20,5\% dos discentes de enfermagem. Além disso, 
11 | Souza fO, Silva RM, Costa ALS, Mussi FC, Santos CCT, Santos OP

11,1\% dos discentes apresentou muito alto estresse relacionado ao Ambiente. Em revisão da literatura conduzida em 2014, nas bases Medical Literature onLine, Cumulative Index to Nursingand Allied Health Literature, Psychology Informationand Pub Med, os estressores identificados em discentes de enfermagem de primeiro ano foram: o ingresso em um curso universitário; o notável volume de atividades em um período curto de tempo; manutenção de boas notas; avaliações e exames; gerenciar as questões financeiras; cometer erros com os pacientes; e aprender procedimentos clínicos. ${ }^{14}$

Tais estressores perpassam os fatores de estresse do AEEE, pois as questões relacionadas a avaliações, testes e notas são descritas no fator Atividades Teóricas; os aspectos financeiros e as transformações vivenciadas quando do ingresso no curso são abordados no fator Ambiente; o Gerenciamento do Tempo envolve questões relativas à dificuldade de administrar a demanda acadêmica para que seja possível atender as necessidades sociais, familiares e pessoais; e as preocupações com aspectos clínicos, como procedimentos e manejo do paciente, relacionam-se à Realização de Atividades Práticas. ${ }^{2}$ Dessa maneira, os estudos anteriores ${ }^{2,14}$ com alunos ingressantes confirmam a presença dos estressores aqui encontrados. Por essa razão, pesquisadores recomendam melhorias no escopo dos currículos de enfermagem, com base nos estressores evidenciados na literatura, o que inclui: considerar o tempo necessário para a realização das atividades propostas a fim de evitar sobrecarga; e utilizar formas alternativas de avaliação que vão além de testes de aquisição de habilidades e conhecimentos esperados. ${ }^{14}$

$\mathrm{Na}$ análise dos níveis de estresse psicoemocional, observou-se predomínio de discentes com médio nível de estresse, seguido por aqueles que relataram alto estresse. Por meio do Student-Life Stress Inventory, pesquisa com 283 universitários da área de saúde do Paquistão, incluindo estudantes de enfermagem, verificou predomínio de alto estresse nos estudantes de odontologia, medicina e enfermagem, sendo os escores superiores ao limite definido pelo instrumento. $^{7}$ 
Estresse e resiliência em discentes de enfermagem de duas universidades públicas paulistas | 12

O estresse impacta negativamente na saúde física e mental do discente e pode levar à Síndrome de Burnout, à baixa qualidade do sono, ao ganho de peso, ao absenteísmo por doença e à desistência do curso. Nesse sentido, além da revisão da estrutura curricular tradicional, sugere-se que os gestores das instituições de ensino considerem a possibilidade de avaliações regulares dos discentes para identificação precoce de desordens psicológicas, especialmente aquelas decorrentes do processo de formação. ${ }^{15}$ A partir dessas informações, seria possível desenvolver e aplicar intervenções focadas na necessidade do aluno, tais como o aconselhamento individual, o fortalecimento de estratégias de enfrentamento dos estressores e a promoção da personalidade resiliente. ${ }^{16}$

Não foi observada correlação significativa entre os escores de estresse (geral e por domínio) e os níveis de resiliência entre os discentes de enfermagem avaliados. Ainda, houve predomínio de reduzidos níveis de resiliência entre os discentes, seguido por níveis moderados, sendo o domínio Ações e Valores o que mais contribuiu para a resiliência na amostra. Em pesquisa com 275 estudantes de medicina de uma universidade privada de São Paulo, identificou-se o predomínio de moderada resiliência em todos os anos do curso e na avaliação geral da população. ${ }^{17}$ Os achados dessa pesquisa e do estudos com estudantes de medicina demonstram a presença de discentes com moderada resiliência, o que indica que esses indivíduos têm desenvolvido e fortalecido as características resilientes a partir da interação com as adversidades típicas da formação acadêmica dos cursos da área de saúde, como provas, cuidado a outros seres humanos, a convivência com a morte e as próprias exigências mercadológicas da profissão. ${ }^{17-18}$ Além disso, é importante considerar aquelas dificuldades enfrentadas no âmbito pessoal, familiar e laboral, os quais são vivenciados no espaço externo às instituições de ensino, mas que mobilizam e fortalecem os recursos de enfrentamento dos estudantes de forma geral. ${ }^{17-19}$ 
Nesse contexto, a partir desse enfrentamento frequente de adversidades, as ações e valores (domínio do constructo de resiliência) já apresenta escores superiores aos demais. Ele refere-se às ações relacionadas a energia, persistência, disciplina e concepção de valores que, quando executados, dão sentido à vida, como a amizade, a realização pessoal e a satisfação ${ }^{9-10}$ Nesse sentido, ao perceber os potenciais estressores como desafios necessários e importantes ao desenvolvimento e aprendizado, os discentes passam a compreender os estressores acadêmicos e pessoais de maneira positiva e adotam comportamentos e atitudes necessários à adaptação ao ambiente acadêmico, o que leva a menores níveis de estresse, depressão, Burnout, dentre outros, além de melhorar a satisfação com o curso e desempenho acadêmico. Por isso, a resiliência vista como um fator protetor da saúde. ${ }^{17-19}$

Dessa forma, pesquisadores têm proposto o desenvolvimento e aplicação de programas de treinamento das habilidades emocionais, mentais e comportamentais que fortalecem a resiliência em discentes da área de saúde, em especial de enfermagem (por essa ser considerada uma profissão estressante frente as demandas laborais vinculados ao processo de trabalho), os quais já têm sido aplicados em estudantes de ensino médio, ${ }^{18}$ mas ainda precisam ser melhor explorados quanto aos fatores que influenciam a resiliência no contexto específico da formação universitária. ${ }^{17-18}$

Sobre isso, em revisão sistemática conduzida em 2014, com seleção de intervenções cognitivo-comportamentais, como a promoção de personalidade hardiness e resiliente, evidenciou-se a efetividade das mesmas para redução do estresse, ansiedade e sintomas depressivos. ${ }^{16}$ Contudo, os autores apontam a necessidade de amostras heterogêneas e de superação das limitações metodológicas dos estudos experimentais incluídos. ${ }^{16,19}$ Dessa forma, seria possível o planejamento e aplicação de intervenções que minimizem as alterações de saúde nos discentes, com a prevenção de processos crônicos, como o Burnout; bem como da queda no desempenho acadêmico. ${ }^{15}$ 
Estresse e resiliência em discentes de enfermagem de duas universidades públicas paulistas | 14

\section{Conclusão}

Observou-se predominância de médio nível de estresse geral, sendo que o gerenciamento do tempo e as atividades teóricas representaram alto nível de estresse para $23,9 \%$ e $20,5 \%$ da população. Além disso, $11,1 \%$ dos discentes apresentaram muito alto estresse relacionado ao Ambiente. Os discentes de enfermagem apresentaram reduzidos níveis de resiliência, seguido por níveis moderados; e não houve correlação significativa entre o estresse e os níveis de resiliência entre os discentes avaliados.

O ambiente de formação em enfermagem apresenta potencial para o adoecimento do discente, embora parte dos alunos já apresente resiliência moderada. Por isso, é importante que as instituições formadoras proponham ações que permitam um ambiente saudável de formação dentro dos seus espaços e possibilidades. Para isso, a carga de atividades teóricas, as dificuldades com o manejo de tempo entre demandas acadêmicas e pessoais, além das limitações em relação à comunicação profissional, são estressores que podem ser minimizados por meio de ações institucionais, tais como a revisão de elementos curriculares, proposição de espaços para relaxamento e conivência, bem como a criação de espaços, com equipe multiprofissional, para a prevenção de doenças e promoção da saúde dos graduandosexperiência já realizada com sucessos em alguns países desenvolvidos.

Como limitações desse estudo, destacam-se a escassez de estudos, especialmente sobre a resiliência, com discentes de enfermagem, o que dificultou a comparação dos achados dessa pesquisa com outras envolvendo alunos da mesma área. Além disso, essa pesquisa envolveu discentes inserido na realidade de uma grande metrópole e de instituição públicas federais e estaduais. Nesse sentido, sugere-se a realização de novas pesquisas que envolvam discentes de outros cursos da área de saúde, inseridos em espaços privados de formação e em cidades menores. Isso permitirá identificar fatores específicos do processo formativo ou do cotidiano 
que possam interferir nos níveis de estresse e, por conseguinte, na qualidade de vida do discente.

\section{Referências}

1. Reeve KL, Shumaker CJ, Yearwood EL, Crowell NA, Riley JB. Perceived stress and social support in undergraduate nursing student's educational experiences. Nurse Educ Today [Internet]. 2013 [acesso em 2018 fev 01];33(4):419-24. Disponível em: https://www.nurseeducationtoday.com/article/S02606917(12)00382-6/pdf

2. Costa ALS, Polak C. Construção e validação de instrumento para avaliação de estresse em estudantes de enfermagem (AEEE). Rev Esc Enferm USP [Internet]. 2009 [acesso em 2018 fev 01];43(N Esp):1017-26. Disponível em: http://www.scielo.br/pdf/reeusp/v43nspe/a05v43ns.pdf

3. Lazarus RS, Folkman S. Stress, appraisal, and coping. New York (US): Springer; 1984.

4. Bublitz S, Guido LA, Lopes LFD, Freitas EO. Associação entre estresse e características sociodemográficas e acadêmicas de estudantes de enfermagem. Texto \& Contexto Enferm [Internet]. 2016 [acesso em 2018 fev 01];25(4):e2440015. Disponível em: http://www.scielo.br/pdf/tce/v25n4/pt_01040707-tce-25-04-2440015.pdf

5. Yusoff MS, Abdul Rahim AF, Baba AA, Ismail SB, MatPa MN, Esa AR. Prevalence and associated factors of stress, anxiety and depression among prospective medical students. Asian J Psychiatr [Internet]. 2013 [acesso em 2018 fev 01];6(2):128-33. Disponível em: https://www.asianjournalofpsychiatry.com/article/S1876-2018(12)00186-4/fulltext

6. Moreira DP, Furegato ARF. Estresse e depressão entre alunos do último período de dois cursos de enfermagem. Rev Latinoam Enferm [Internet]. 2013 [acesso em 2018 fev 01];21(N Esp):155-62. Disponível em: http://www.scielo.br/pdf/rlae/v21nspe/pt_20.pdf

7. Rehmani N, Khan QA, Fatima SS. Stress, anxiety and depression in students of a private medical school in Karachi, Pakistan. Pak J Med Sci [Internet]. 2018 [acesso em 2018 fev 01];34(3):696-701. Disponível em: https:/www.ncbi.nlm.nih.gov/pmc/articles/PMC6041547/pdf/PJMS-34-696.pdf

8. Wagnild GM, Young HM. Development and psychometric evaluation of resilience scale. J Nurs Meas [Internet]. 1993 [acesso em 2018 fev 01];1:165-78. Disponível em: https://sapibg.org/download/1054wagnild_1993_resilience_scale_2.pdf

10. Pesce RP, Assis SG, Avanci JQ, Santos NC, Malaquias JV, Carvalhaes R. Adaptação transcultural, confiabilidade e validade da escala de resiliência. Cad Saúde Pública [Internet]. 2005 [acesso em 2018 fev 01];21(2):436-48. Disponível em: http://www.scielo.br/pdf/csp/v21n2/10.pdf 
Estresse e resiliência em discentes de enfermagem de duas universidades públicas paulistas | 16

11. Hair Jr JF, Anderson RE, Tatham RL, Black WC. Análise multivariada de dados. $5^{\text {th }}$ ed. Porto Alegre: Bookman; 2005.

12. Benavente SBT, Silva RM, Higashi AB, Guido LA, Costa ALS. Influência de fatores de estresse e características sociodemográficas na qualidade do sono de estudantes de enfermagem. Rev Esc Enferm USP [Internet]. 2014 [acesso em 2018 fev 01];48(3):514-20. Disponível em: http://www.scielo.br/pdf/reeusp/v48n3/pt_0080-6234-reeusp-48-03-514.pdf

13. Bublitz S. Estresse em discentes de enfermagem de quatro instituições brasileiras [dissertação]. Santa Maria: Universidade Federal de Santa Maria; 2014. 89 p.

14. Alzayyat A, Al-Gamal E. A review of the literature regarding stress among nursing students during their clinical education. Int Nurs Rev [Internet] 2014 [acesso em 2018 fev 01]:61(3):406-15. Disponível em: https://onlinelibrary.wiley.com/doi/pdf/10.1111/inr.12114

15. Goel AD, Akarte SV, Agrawal SP, Yadav V. Longitudinal assessment of depression, stress, and burnout in medical students. J Neurosci Rural Pract [Internet]. 2016 [acesso em 2018 fev 01];7(4):493-8. Disponível em: https://www.ncbi.nlm.nih.gov/pmc/articles/PMC5006458/

16. Davies EB, Morriss R, Glazebrook C. Computer-delivered and web-based interventions to improve depression, anxiety, and psychological well-being of university students: a systematic review and metaanalysis. J Med Internet Res [Internet]. 2014 [acesso em 2018 fev 01];16(5):e130. Disponível em: https://www.ncbi.nlm.nih.gov/pmc/articles/PMC4051748/

17. Martinez JE, Pereira DA, Barril ES, Matos SF, Santos RM. Resiliência em estudantes de medicina ao longo do curso de graduação. Rev Fac Ciênc Med Sorocaba. 2016;18(1):15-8.

18. Ungar M, Russell P, Connelly G. School-Based interventions to enhance the resilience of students. J Educ Develop Psychol. 2014;4(1):1-18.

19. Cruz EJER, Souza NVDO, Amorim LKA, Pires AS, Gonçalves FGA, Cunha LP. Resilience as an object of study of occupational health: narrative review. Rev Pesqui Cuid Fundam [Internet]. 2018 [acesso em 2018 fev 01];10(1):283-8. Disponível em: http://www.seer.unirio.br/index.php/cuidadofundamental/article/view/5047/pdf

\section{Autor correspondente}

Rodrigo Marques da Silva

E-mail: marques-sm@hotmail.com

Endereço: Conjunto Residencial 7 Condomínio 1 Bloco D 88. "Apto 1004- Condominio Parque Clube 2". Parque das Cachoeiras, Valparaíso de Goiás, Goiás, Brasil.

CEP: 72872704 
17 | Souza fO, Silva RM, Costa ALS, Mussi FC, Santos CCT, Santos OP

\section{Contribuições de Autoria}

\section{1 - Fernando Oliveira de Souza}

Contribuições: Construção do projeto, condução da coleta e análise dos dados, bem como revisão crítica da versão final do relatório de pesquisa.

\section{2 - Rodrigo Marques da Silva}

Contribuições: Construção do projeto, condução da coleta e análise dos dados, bem como revisão crítica da versão final do relatório de pesquisa.

\section{3 - Ana Lúcia Siqueira Costa}

Contribuições: Suporte e orientação na construção do projeto, condução da coleta e análise dos dados, bem como revisão crítica da versão final do relatório de pesquisa.

\section{4 - Fernanda Carneiro Mussi}

Suporte e orientação na construção do projeto, condução da coleta e análise dos dados, bem como revisão crítica da versão final do relatório de pesquisa.

\section{5 - Carla Chiste Tomazoli Santos}

Suporte e orientação na construção do projeto, condução da coleta e análise dos dados, bem como revisão crítica da versão final do relatório de pesquisa.

\section{6 - Osmar Pereira dos Santos}

Suporte e orientação na construção do projeto, condução da coleta e análise dos dados, bem como revisão crítica da versão final do relatório de pesquisa.

\section{Como citar este artigo}

Souza FO, Silva RM, Costa ALS, Mussi FC, Santos CCT, Santos OP. Estresse e resiliência em discentes de enfermagem de duas universidades públicas paulistas. Rev. Enferm. UFSM. 2019 [Acesso em: Anos Mês Dia];vol.10 e2: 1-17. DOI:https://doi.org/10.5902/2179769234162 